\title{
Tsafon
}

Revue d'études juives du Nord

$74 \mid 2017$

La Déclaration Balfour, vers l'État d'Israël ?

\section{La Déclaration Balfour}

Denis Charbit

\section{OpenEdition}

Journals

Édition électronique

URL : https://journals.openedition.org/tsafon/394

DOI : $10.4000 /$ tsafon.394

ISSN : 2609-6420

\section{Éditeur}

Association Jean-Marie Delmaire

\section{Édition imprimée}

Date de publication : 1 décembre 2017

Pagination : $31-38$

ISSN : 1149-6630

\section{Référence électronique}

Denis Charbit, « La Déclaration Balfour », Tsafon [En ligne], 74 | 2017, mis en ligne le 31 mai 2018, consulté le 24 juin 2021. URL : http://journals.openedition.org/tsafon/394 ; DOI : https://doi.org/ 10.4000/tsafon.394 


\section{La Déclaration Balfour}

\section{Denis Charbit*}

Trois paragraphes, vingt lignes, cent vingt-huit mots : jamais dans les annales de la diplomatie européenne un texte aussi court n'aura eu autant de conséquence sur l'avenir politique d'une région du monde. Grâce à cette déclaration, le nom de Balfour est passé à la postérité. Ni ses essais philosophiques, ni son leadership au sein du parti conservateur britannique, pas plus que sa gestion des affaires en Irlande comme secrétaire d'État ou son œuvre législative en matière d'éducation comme député à la Chambre des Communes, n'ont laissé de trace impérissable. Premier ministre de janvier 1902 à décembre 1905, quand bien même c'est sous son règne qu'a été scellée l'Entente cordiale entre la France et le Royaume-Uni, son action à la tête du gouvernement a été éclipsée par la Déclaration qui porte son nom, datée du 2 novembre 1917, avec en-tête du Foreign Office puisqu'il en était alors le titulaire entre 1916 et 1919. Cette notoriété ne lui valut pas que de la gratitude : s'il a joui d'un grand prestige auprès des Juifs sionistes - on trouve des rues Balfour dans la plupart des localités israéliennes - il a été, pour les Arabes de Palestine et les musulmans, celui par qui le scandale et le malheur arrivent.

Que dit-elle en substance? Outre la bienveillance exprimée en faveur de « l'établissement en Palestine d'un foyer national pour le peuple juif », le gouvernement britannique se déclare disposé à apporter ses efforts « pour faciliter la réalisation de cet objectif ».

\footnotetext{
* Université ouverte d'Israël, Raanana.

Cet article est paru dans Abdelwahab Meddeb et Benjamin Stora (dir.), Histoire des relations entre juifs et musulmans, des origines à nos jours, Paris, Albin Michel, 2013, pp. 320-328. Nous remercions les éditions Albin Michel d'avoir autorisé Tsafon à le publier de nouveau.
} 
Les mots ont été pesés et choisis avec prudence. Ils pourront toujours être interprétés par les autorités britanniques dans un sens restrictif ou extensif, le sort est jeté, au grand dam des nationalistes arabes qui s'estiment floués, à la grande satisfaction de la direction sioniste qui prend la juste mesure du bond en avant historique qui vient d'être accompli. Certes, un «foyer national» n'est pas un État, mais le projet a été entériné et sa forme juridique définitive peut attendre ; pour la première fois, dans un texte aux implications politiques évidentes, la collectivité juive a été définie comme un peuple - ce qui n'allait pas de soi à l'époque, y compris pour nombre de juifs attachés à une définition exclusivement religieuse de leur particularisme identitaire; s'il est précisé que c'est bien en Palestine que ce foyer sera établi, à l'exclusion de tout autre territoire, ce n'est pas nécessairement sur toute la Palestine, dont il faut encore fixer les frontières, qu'il pourra s'étendre; enfin, les deux concessions sommaires et formelles stipulant que « rien ne sera fait qui portera préjudice aux droits civils et religieux des populations [autochtones] non juives en Palestine » comme aux droits politiques et au statut juridique des juifs dans tous les pays où ils sont établis, n'altèrent nullement la consécration diplomatique capitale octroyée au sionisme.

Presque cent ans après sa promulgation, la controverse ne s'est toujours pas refroidie. Dans l'histoire du conflit écrite en parallèle par un collectif d'enseignants livrant sur chaque double page le narratif respectif qu'Israéliens et Palestiniens se racontent, l'écart reste considérable ${ }^{1}$. La lettre adressée au dirigeant de l'organisme représentatif de la communauté juive de Grande-Bretagne, Lionel de Rothschild, témoigne, pour les premiers, de la sympathie qu'Arthur James Balfour éprouva pour une cause généreuse compatible avec les intérêts britanniques, réparant ainsi une injustice historique. Héritière de l'Empire romain qui avait soumis le royaume juif à son autorité, détruit le Second Temple et débaptisé la Judée pour la nommer Palestine, la Grande-Bretagne faisait, dix-huit siècles et demi plus tard, amende honorable. Les sionistes laïques la tiennent pour la Magna Carta de leur cause ${ }^{2}$ tandis que les sionistes religieux, sensibles à la répétition de l'Histoire lorsque le modèle initial est d'origine biblique, voient dans la Déclaration Balfour la

\footnotetext{
${ }^{1}$ Voir Apprendre le narratif historique de l'Autre. Palestiniens et Israéliens, Beit Jallah, éditions PRIME (Peace Research Institute in the Middle East), 2003, pp. 6-10 (en hébreu). Le livre a été traduit en français par Rosie Pinhas-Delpuech sous le titre Histoire de l'autre, Paris, Liana Levi, 2004.

2 Simha Flapan, The Birth of Israel: Myths and Realities, Pantheon Books, New York, 1987, p. 16.
} 
réédition du décret de l'empereur perse Cyrus en -526 autorisant les Juifs exilés sur les rives de Babylone après la destruction du Premier Temple à revenir en Judée pour y restaurer leur indépendance. Pour les Arabes, et en premier lieu les Palestiniens, la déclaration Balfour demeure l'expression la plus arbitraire de l'impérialisme occidental, la négation la plus manifeste de leur existence puisque c'est leur identité propre et leurs droits politiques et nationaux qu'on assassine : ils ne sont désignés que comme des populations non juives ne pouvant prétendre qu'à jouir de droits civils et religieux. La Déclaration Balfour ne fut ni la première ni la dernière intervention de la Grande-Bretagne dans la région, mais elle constitue le «péché originel » irrémissible : à la différence du sort de tant d'autres décisions britanniques dont les Arabes se sont accommodés ou débarrassés, tous les efforts déployés pour réduire la Déclaration Balfour à néant, pour en dévier au moins le cours et en diminuer la portée, ne sont jamais parvenus à inverser la dynamique qu'elle avait suscitée. C'est qu'elle a propulsé et privilégié un nouvel acteur politique - les sionistes porteur de revendications concurrentes aux leurs.

Pour le mouvement sioniste, la Déclaration Balfour inaugure sans aucun doute une nouvelle ère, celle de "L'État en marche », mais elle correspond aussi à l'aboutissement d'une stratégie diplomatique que le père du nationalisme juif, Theodor Herzl, avait élaborée durant ses dix années d'activité sioniste. Après avoir vainement prié les philanthropes juifs d'éponger les dettes de l'Empire ottoman en contrepartie d'une concession territoriale en Palestine que le sultan leur aurait accordée, Herzl avait estimé que le seul moyen de concrétiser la «solution moderne » d'un État pour les Juifs était d'obtenir le concours d'une grande puissance. Celle-ci proclamerait une Charte en faveur du mouvement sioniste, c'est-à-dire fournirait sur la scène internationale un appui moral, politique et financier à la reconstruction d'un foyer national juif en Palestine. Les démarches entreprises en ce sens par Herzl jusqu'à sa disparition étaient demeurées sans effet: ni l'empereur allemand Guillaume II avec lequel il obtint une rapide entrevue lors de sa visite officielle en Palestine ni le ministre de l'Intérieur Plehve auprès du tsar Nicolas II ne prirent au sérieux sa proposition. Seul le ministre des Colonies britannique, soucieux d'éviter un nouvel afflux incontrôlé d'immigrants juifs consécutif au pogrom de Kichinev, conçut en 1904 le projet de les détourner vers l'Ouganda et de créer, à cet effet, une république juive sous la houlette d'Herzl. L'offre n'alla pas plus loin, mais précipita le mouvement sioniste au bord du schisme. Détourner les yeux 
de Sion était une infidélité coupable, sinon une trahison. Herzl, épuisé, mourut quelques mois plus tard. La controverse eut cependant l'insigne mérite de clarifier une fois pour toutes un débat interne au mouvement sioniste à l'issue duquel il fut décrété que, désormais, il n'y aurait « pas de sionisme sans Sion»: seul un territoire situé dans la région correspondant peu ou prou à la Palestine antique devait être retenu pour y rassembler les Juifs. La stratégie diplomatique n'étant guère couronnée de succès, les partisans du sionisme pratique préconisèrent l'acquisition de terres et leur mise en valeur, parcelle par parcelle, lopin de terre par lopin de terre, plutôt que de placer leurs espoirs dans un improbable soutien diplomatique qui tardait à venir. Écartant ces vaines querelles entre courants sionistes mus par le même objectif, Haïm Weizmann plaida pour un " sionisme synthétique » : la clé du succès de l'entreprise sioniste repose sur la conjugaison des efforts tant sur le terrain que dans l'arène internationale.

La déclaration solennelle n'était pas seulement une reconnaissance de principe. Elle s'inscrivait dans un contexte propice à lui fournir une signification politique opérationnelle et une valeur performative imminente. L'Empire ottoman s'étant rangé du côté de l'Allemagne dès novembre 1914, les Britanniques avaient décidé d'ouvrir un front en Orient, estimant que la zone était le maillon faible de la défense allemande : pour rompre le rapport de forces militaire en Europe paralysé par la guerre des tranchées, il fallait convaincre les États-Unis de mettre leur poids dans la balance et, en attendant leur revirement, ouvrir un autre front contre l'Allemagne et l'Empire austro-hongrois en remontant du Moyen-Orient jusqu'aux Balkans. Ces calculs militaires visant à hâter la victoire n'étaient pas dépourvus de considérations stratégiques à long terme : pour sécuriser les positions britanniques situées de part et d'autre de l'Orient - en Égypte et en Inde - et consolider leur empire dans la région, il était bien utile de s'emparer du Moyen-Orient. Pour le mouvement sioniste, la Première Guerre mondiale rétablissait la priorité de l'action diplomatique et la nécessité de faire des choix. Le premier fut de rompre avec la neutralité qui avait été proclamée avec le déclenchement des hostilités. Weizmann pariait pour la victoire de la Grande-Bretagne et dès lors que celle-ci définissait comme but de guerre le démantèlement de l'Empire ottoman, il importait de se placer sous son aile protectrice dans l'espoir d'être invité à la table des vainqueurs. Associé à la rédaction de la Déclaration, Weizmann pouvait se prévaloir d'un résultat qui accomplissait la stratégie préconisée par Herzl. En 
déclarant la guerre à l'Empire ottoman, les Britanniques offraient aux nationalistes arabes tout autant matière à espérer: la Révolution des Jeunes Turcs en 1908 ayant suscité de vains espoirs d'autonomie, ils pouvaient désormais entrevoir la réalisation de leurs aspirations nationales avec la suppression de l'obstacle ottoman qui fut leur premier adversaire. En échange d'une participation, fût-elle symbolique, aux grandes manœuvres militaires en préparation, Juifs et Arabes achetaient leur droit d'entrer dans la danse des grandes manœuvres diplomatiques. Le scénario était limpide : le coup de grâce militaire entraînerait l'agonie, définitive cette fois, de «l'homme malade de l'Europe », d'où naîtrait, outre la République turque indépendante réduite à son sanctuaire territorial par le traité de Sèvres du 10 août 1920, révisé en 1923, une création politique inédite destinée à fixer le sort des provinces arabes de l'ex-Empire ottoman moribond. Quelle forme devait-elle prendre ? Un État ou plusieurs, une fédération ou des protectorats ? La réponse était déterminante pour les Arabes, secondaire pour les sionistes qui souhaitaient avant tout être associés aux destinées d'un territoire en Palestine. Concilier les appétits de puissance britanniques, les prétentions françaises, les aspirations nationales juives et les revendications autochtones arabes, tel fut l'enjeu de cette décennie capitale pour le Moyen-Orient : entre 1914 et 1923 les provinces arabes allaient connaître leur plus formidable mutation politique depuis l'avènement en 1453 de l'Empire ottoman sous l'autorité duquel elles avaient été jusque-là placées.

C'est à la veille de cette grande redistribution territoriale, en vue de ce grand découpage historique, que la puissance britannique publia la Déclaration Balfour, offrant à la communauté juive de Palestine et à la direction du mouvement sioniste un traitement de faveur qui était loin d'être acquis. Côté britannique, la Déclaration Balfour répondit à plusieurs intérêts. Soulevée dès 1915, la proposition figurait dans maints rapports et mémorandums soumis au gouvernement et recueillait des avis favorables inspirés par des motifs religieux. La conjoncture allait transformer une inclination émouvante en décision politique effective : préoccupés de convaincre les États-Unis d'entrer en guerre aux côtés des Alliés, les Britanniques se heurtaient à un courant isolationniste fort répandu parmi les Américains, y compris les Juifs. Originaires de Russie pour la plupart, ces derniers ne pouvaient admettre que leur nouvelle patrie s'alliât avec le tsar qu'ils vouaient aux gémonies à cause de sa politique antisémite qui les avait poussés à l'exil et plongeait encore dans 
la misère leurs frères demeurés en Russie. Comme il était exclu d'intervenir dans les affaires intérieures du tsar, c'est en apportant son soutien à la cause sioniste que la Grande-Bretagne pouvait escompter un changement d'attitude de la part des Juifs américains. La révolution russe de février 1917 ne changea rien à la pertinence d'une déclaration opportune, bien au contraire : les Britanniques redoutaient la tentation russe d'une paix séparée avec l'Allemagne. Un appui manifeste au sionisme pourrait inciter les Juifs de Russie à encourager le gouvernement révolutionnaire provisoire à poursuivre la guerre. Outre l'impact sur l'entrée en guerre des Américains et le maintien de la Russie dans le camp des Alliés, en appuyant le développement d'un foyer national juif en Palestine, les Britanniques entendaient surtout damer le pion aux Français. N'avaient-ils pas été les premiers à exprimer leur volonté «d'aider à la renaissance [...] de la nationalité juive, sur cette terre d'où le peuple d'Israël a été chassé il y a tant de siècles ? » Adressé à Nahum Sokolov, le 4 juin 1917, par Jules Cambon, secrétaire aux Affaires étrangères, le message diplomatique, qui s'achevait sur un engagement solennel (« Le gouvernement français [...] ne peut éprouver que de la sympathie pour votre cause dont le triomphe est lié à celui des alliés. Je suis heureux de vous en donner ici l'assurance ») $)^{3}$, fut interprété comme un avertissement: quatre mois plus tard, le gouvernement britannique publiait la Déclaration Balfour d'inspiration similaire afin de barrer la route aux visées françaises sur la Palestine. Enfin, il importait d'être en phase avec l'évolution sur le terrain en dotant d'une légitimité morale l'occupation militaire imminente de la Palestine: la conquête reposerait non sur la force mais le droit, non sur un caprice impérialiste, mais sur un projet d'autodétermination éminemment respectable conforme aux nouvelles normes internationales définies par le président Wilson pour l'après-guerre. Un mois et une semaine après la Déclaration Balfour, le 11 décembre, le commandant des forces armées de sa Majesté, le général Allenby, entrait dans Jérusalem. Un an plus tard, le 7 décembre 1918, le cessez-le-feu était signé.

La Déclaration Balfour n'était pas le seul projet relatif à l'avenir de la Palestine. Elle était même la dernière en date des promesses contractées par les Britanniques pendant la guerre. Deux autres l'avaient précédée : la première avait été faite par le gouverneur britannique de l'Égypte, Henry McMahon au chérif Hussein, rejeton de la prestigieuse

\footnotetext{
${ }^{3}$ Voir Renée Neher-Bernheim, La Déclaration Balfour, Paris, Julliard, coll. Archives, 1969 , pp. 254-255.
} 
dynastie hachémite remontant à Mahomet. Un musulman s'alliait à un chrétien pour combattre d'autres musulmans: le précédent était spectaculaire d'autant que le musulman en question était le protecteur de La Mecque et Médine. En contrepartie d'une insurrection générale victorieuse contre l'Empire ottoman, il obtiendrait un grand royaume autonome ${ }^{4}$. Dans la première lettre, McMahon resta évasif sur la question des frontières dudit royaume concédé à Hussein. Devant son insistance, McMahon finit par obtempérer, mais plutôt que d'envoyer une carte précise, voire de désigner par écrit les régions qui y seraient intégrées, il dressa la liste des territoires qui en seraient exclus: à l'est, la Mésopotamie (correspondant à l'Irak actuel), au nord-ouest, la région de Mersin et d'Alexandrette, le littoral syrien et libanais de Lattaquié à Beyrouth, et « la zone syrienne située à l'ouest des districts de Damas, de Homs, de Hama et d'Alep ». La Palestine ne fut pas mentionnée comme telle, ouvrant ainsi une boîte de Pandore : étant au sud-ouest de la Syrie, est-elle en dehors du royaume ou en fait-elle partie?

La seconde promesse n'était pas une offre proposée à un parti tiers, mais des accords secrets dûment signés en mars 1916, avec cartes à l'appui cette fois, par la Grande-Bretagne et la France pour se partager le butin de guerre en cas de victoire. Les négociateurs, Mark Sykes (du Foreign Office) et Georges Picot (du quai d'Orsay), avaient établi sur une carte du Levant une première distinction entre zones de contrôle et zones d'influence en fonction du type de domination, rigide ou plus lâche, que les deux puissances entendaient y exercer. Elles s'étaient également entendues sur la mise en place d'un condominium international, principalement franco-britannique, sur la Palestine, Jérusalem et les Lieux saints.

La correspondance Hussein-McMahon, les accords Sykes-Picot et la Déclaration Balfour avaient été élaborés en temps de guerre pour répondre à des pressions et des intérêts politiques conjoncturels. Une fois les hostilités terminées, les Alliés, vainqueurs déclarés de la guerre, pouvaient passer aux actes et tirer parti de leur victoire si ce n'est que le contexte était quelque peu différent de ce qui avait été pressenti : les Français n'avaient pas participé aux combats, ce qui réduisait leurs prétentions à la baisse; plongés dans une guerre civile face aux Russes blancs et dans une guerre tout court contre la coalition internationale qui s'était constituée contre eux, les Bolcheviks, préoccupés de conserver

\footnotetext{
${ }^{4}$ Il s'agit de dix lettres que les deux hommes se sont échangées entre juillet 1915 et mars 1916. L'ensemble est connu sous le nom de « Correspondance Hussein-McMahon ».
} 
leur pouvoir, étaient hors-jeu; les Américains s'étaient de nouveau repliés sur leur isolationnisme; la Grande-Bretagne était la seule vraie puissance sur le terrain. Elle n'en était pas moins tenue de gérer et d'harmoniser, autant que faire se peut, les revendications concurrentes présentées par les sionistes, les nationalistes arabes et la diplomatie française.

Weizmann protesta contre les accords Sykes-Picot qui divisaient le territoire de la Palestine en quatre zones, destinées respectivement à la France, à la Grande-Bretagne, au royaume arabe et, enfin, à une autorité internationale conjointe ; Hussein s'en prit, lui, à la Déclaration Balfour qui amputait le royaume arabe à venir de la Palestine et de Jérusalem, troisième ville sainte de l'Islam.

Cependant, si la dynastie hachémite n'était guère indifférente au sort de la Palestine pour des raisons religieuses bien plus que politiques, l'objectif primordial de l'émir Hussein et ses fils, en tant que chefs de file du nationalisme arabe, était l'avènement du grand royaume promis par McMahon. Dans un premier temps, les Britanniques semblaient s'y résoudre : jouissant de leur soutien, l'émir Fayçal se rendit à Damas en juin 1918 pour établir son autorité in situ. Pour les Arabes de Palestine, entre la Déclaration Balfour et un royaume arabe en gestation, le choix était fait : le 2 novembre 1918, ils marquaient le premier anniversaire de la Déclaration britannique par une manifestation de masse suivie d'une pétition au gouvernement de sa Majesté signée par les édiles et les notables pour réclamer l'intégration de la Palestine dans le giron syrien puisqu'elle en constituait le flanc méridional, ainsi qu'ils le déclaraient à l'envi. À cet égard, les germes d'un conflit potentiel étaient semés. Mais soucieux de tenir compte des aspirations sionistes, l'émir Fayçal signa le 3 janvier 1919 sous les auspices du colonel Lawrence un accord de coopération mutuelle avec Weizmann, incluant une délimitation des frontières entre le royaume arabe et le foyer national juif. Il n'appartient pas de dire si la création d'un grand royaume arabe amputé de la seule Palestine aurait atténué sinon évité le conflit, changeant dès lors le destin de la région. Une occasion a été sans doute manquée, une chance de paix a peut-être été compromise. En revenant au mois de septembre 1919 sur la décision de soutenir le règne de Fayçal en Syrie pour s'attribuer des mandats sur les provinces de l'ex-Empire ottoman, l'Histoire - autrement dit, la Grande-Bretagne, de concert avec la France - en a décidé différemment. 